\title{
Bariatric Surgery and the Neuro-Ophthalmologist
}

\author{
Heather E. Moss, MD, PhD ${ }^{\star}, 1,2$ \\ ${ }^{1}$ Department of Ophthalmology \& Visual Sciences, University of Illinois at Chicago, Chicago, IL, \\ USA \\ ${ }^{2}$ Department of Neurology \& Rehabilitation, University of Illinois at Chicago, Chicago, IL, USA
}

\begin{abstract}
Background-As the prevalence of obesity increases, so are the prevalences of weight related diseases and the incidence of surgical procedures to promote weight loss. It is important for neuroophthalmologists to be familiar with these procedures and possible downstream effects on afferent and efferent visual function.
\end{abstract}

Evidence acquisition-Review of ophthalmology, neurology, general surgery, obesity, endocrinology, nutrition, psychiatry and neurosurgery literature.

Results-Bariatric surgery is a safe and effective treatment for weight loss in obese individuals. There is level IV evidence that it is associated with improvement in idiopathic intracranial hypertension(IIH). Laboratory nutrient deficiencies are common following some types of bariatric procedures. Symptomatic deficiencies are less common but can be devastating. Thiamine deficiency can cause nystagmus and other symptoms in weeks to months following surgery, B12 or copper deficiency can cause optic neuropathy in the years to decades following bariatric surgery.

Conclusions-Bariatric surgery may be a treatment for IIH. Postoperative vitamin deficiencies may present with nystagmus, optic neuropathy, nyctalopia and/or ophthalmoparesis weeks to years after surgery.

\section{Keywords}

Bariatric surgery; Nutrient deficiency; Optic neuropathy; Idiopathic intracranial hypertension; Obesity

\begin{abstract}
Bariatric surgery, in which the gastrointestinal (GI) tract is surgically manipulated with the goal of decreasing caloric absorption and ultimately causing weight loss, is relevant to neuroophthalmologists because it is a treatment option we can discuss with our patients who have weight-associated neuro-ophthalmic disease and because neuro-ophthalmic symptoms and signs may result from the nutrient deficiencies that can accompany altered caloric absorption. A challenge in reviewing the relevant literature on bariatric surgery is that it spans numerous specialties, including ophthalmology, neurology, general surgery, obesity,
\end{abstract}

\footnotetext{
*Corresponding author: Department of Ophthalmology (MC 648), 1855 W Taylor St. 3.158, Chicago, IL 60612, 312-996-9120, hemoss@uic.edu.

Conflict of interest: Dr. Moss has no disclosures related to this manuscript
} 
endocrinology, nutrition, psychiatry and even neurosurgery. Furthermore, there is heterogeneity in the literature since bariatric surgery encompasses multiple distinct surgical procedures and some of these procedures are also used for non-bariatric indications. Another barrier is the relative rarity of the associated neuro-ophthalmic conditions, which makes both definitive epidemiological and treatment studies challenging.

\section{Bariatric Surgery - who gets it?}

Guidelines for the management of obesity, most recently published by the American College of Cardiology, American Heart Association and the Obesity Society,[1] recommend consideration of bariatric surgery in patients who are motivated and who have not responded to behavioral weight loss treatment (with or without pharmacotherapy) based on body mass index $\left(\mathrm{BMI}=(\right.$ weight in $\left.\mathrm{kg}) /(\text { height in } \mathrm{m})^{2}\right)$ and the presence of weight-related comorbidities. The two categories warranting consideration are BMI $\geq 40 \mathrm{~kg} / \mathrm{m}^{2}$ or BMI $\geq$ $35 \mathrm{~kg} / \mathrm{m}^{2}$ with a weight related comorbidity.

What is considered to be a comorbidity varies from study to study. The most widely referenced bariatric surgery outcome scale includes hypertension, cardiovascular disease, type II diabetes, sleep apnea, obesity/hyperventilation syndrome, osteoarthritis and infertility as major comorbid conditions. In this scale, idiopathic intracranial hypertension(IIH), venous stasis, gastro-esophageal reflux disease and urinary stress incontinence are considered to be minor comorbid conditions.[2] A more recent scale, which also includes $\mathrm{IIH}$, does not distinguish between major and minor comorbid conditions and is administered based on patient report.[3] This scale incorporates severity of each comorbidity using an integer rating scale from 0 to 5 , where 0 means no symptoms, 1 or 2 means symptoms and 3 to 5 mean diagnosis with varying levels of treatment.[3] Consequently, a person can have a rating of 2 for IIH by reporting headaches even if no other testing has been performed. How the severity of any comorbid conditions might impact surgical decision-making is not yet clear.

Though classified as a weight-related comorbidity in the bariatric surgery literature, IIH remains a relatively rare disease in this population. In a prospective evaluation of comorbidities (assessed by interview) in 226 candidates for gastric bypass surgery, only $1(0.4 \%)$ reported a diagnosis of IIH, while 201 had psychosocial impairment, $173 \mathrm{had}$ musculoskeletal disease, 106 had hypertension and 50 had diabetes.[3] Two studies have evaluated the prevalence of IIH in patients undergoing bariatric surgery evaluation using a protocol of non-mydriatic fundoscopic photographic screening followed by full neuroophthalmic evaluation and appropriate clinical work up for those with photographic abnormalities. Krispel et al. [4] reported 7(0.6\%) of 1148 screened subjects had a history of IIH. 17 of 606 subjects with interpretable photograph (none with a history of IIH) had possible optic disc edema and 4/11 had confirmed mild papilledema with normal vision on subsequent examination. 3 patients had complete work up with lumbar puncture and opening pressures of 24, 25 and $32 \mathrm{~cm} \mathrm{H}_{2} \mathrm{O}$, respectively. The authors concluded that IIH was present in this population with a prevalence of 3/606 (0.5\%), though this excluded 7 subjects with suspicious photographs who could not be completely followed up. In a similar study, Hamdallah et al. reported 4 (0.4\%) of 1084 screened subjects had a prior diagnosis of IIH. 
[5] 16 of 532 subjects with interpretable photographs had possible optic disc edema and 27 had other ophthalmic findings. 4/11 had Frisén stage 1 disc edema on clinical exam and IIH was subsequently diagnosed in 3 (further specifics not given). Of note, two patients photographed with a prior diagnosis of IIH did not have disc edema. Also, two of the patients newly diagnosed with IIH with papilledema were asymptomatic. This casts doubt on any conclusions that are drawn from studies based solely on a survey of symptoms and patient report.

\section{Bariatric Surgery - what is being done?}

Approximately 113,000 bariatric surgical procedures are performed annually in the U.S.[6] Bariatric surgery achieves weight loss through decreased caloric uptake by the body. This is accomplished through restricted food intake, malabsorption(i.e. bypassing a length of intestine), maldigestion(i.e. less exposure to stomach acids), and combinations thereof. There are multiple surgical techniques in use and these techniques are in constant evolution. Currently used techniques are outlined below and shown schematically in Figure 1.

Adjustable Gastric Band(lap band ${ }^{\circledR}$, the band) involves laparoscopic placement of an adjustable band around the upper stomach. This restricts food intake and facilitates a feeling of satiety, which helps to reduce food consumed by the patient. Neither digestion nor absorption is affected. The band is adjustable via injection of saline through a subcutaneous port and is removable.[7] The hospital stay is short(outpatient or 24 hours) and the incidence of complications is reported to be $1 \%$ (major complications) and $3 \%$ (minor complications) in the hands of an experienced surgeon.[1]

Sleeve Gastrectomy(the sleeve) involves laparoscopic removal of the greater curvature of the stomach to create a sleeve-like pouch. This restricts food intake and alters hormone patterns to promote satiety. It also impacts on digestion of food. The hospital stay is typically 2 days and complication rates range between those of gastric band and gastric bypass procedures.[7]

Roux-en-Y Gastric Bypass surgery(gastric bypass, RGB) involves dividing the GI tract at the stomach and at the jejunum, anastomosing the proximal stomach to the distal jejunum and attaching the removed section(distal stomach and proximal jejunum) approximately $1 \mathrm{~m}$ further down the small intestine. This restricts food intake, alters hormone patterns and decreases absorption in the proximal small intestine.[7] It can be performed laparoscopically, with a complication rate of 4-5\%(major complications) and 2-18\%(all complications).[1]

Biliopancreatic diversion(BPD) with or without duodenal switch (DS) involves the creation of a tubular stomach(similar to sleeve gastrectomy) and dividing the gastrointestinal tract at two points, specifically the proximal duodenum and the ileum. The stomach is anastomosed to the ileum and the bypassed section(consisting of $3 / 4$ of the small intestine) is anastomosed to the ileum distal to this. The procedure can be performed laparoscopically. BPD restricts food intake, alters hormone patterns, alters digestion, and substantially decreases absorption.[7] Perioperative complication rates range from 2-8\%.[1] 
Guidelines for the perioperative nutritional, metabolic and non-surgical support of bariatric surgery patients were last published in 2013 by the American Association of Clinical Endocrinologists, the Obesity Society, and the American Society for Metabolic \& Bariatric Surgery.[8] These include recommendations for pre-operative psychosocial, nutritional and cardiopulmonary assessment. Post-operative meal initiation should be supervised by a registered dietitian and patients should receive counseling regarding appropriate food intake. Patients having undergone a gastric banding should receive supplementation with a multivitamin with minerals (including iron, folate and thiamine), calcium and vitamin D. In addition, patients status post other procedures should receive a second daily multivitamin with minerals and vitamin $B_{12}$. Patients status post BPD should also receive zinc supplementation. Supplementation can be oral with the possible exception of vitamin $\mathrm{B}_{12}$, which may require subligual, parenteral, intramuscular or subcutaneous formulations to ensure adequate absorption. Vitamin $\mathrm{B}_{12}$ and urinary calcium should be monitored in all patients. In addition folate, iron, parathormone (parathyroid hormone) and vitamin D should be monitored after RGB and BPD, and vitamin A should be monitored after BPD. Thiamine, selenium, zinc and copper should be evaluated based on clinical findings (e.g. rapid weight loss, vomiting, or symptoms attributable to deficiency). However, studies show that in practice, testing does not meet these guidelines. One study based on claims analysis found that only $50 \%$ of patients had been tested for vitamin $\mathrm{B}_{12}$ and iron status post-operatively.[9] The practice of vitamin supplementation also varies widely.[10]

\section{Bariatric Surgery Outcomes}

\section{Weight loss}

Patients typically achieve weight loss of 20-35\% 2-3 years after surgical procedures, this degree of weight loss being 14-37\% greater than comparable non-surgical cohorts.[1] Evidence for longer-term success rates is less strong because there is a tendency for some weight to be regained by 10 years. Studies suggest that patients exhibiting similar weight loss after 2-3 years do better at 10 years following gastric bypass than gastric banding.

\section{Idiopathic Intracranial Hypertension}

Fridley et al. recently published a meta-analysis of 62 patients ( 11 case series and case reports) who had IIH and underwent bariatric surgery.[11] Lumbar puncture opening pressure was reported in 55 patients, ranging from $20 \mathrm{~cm} \mathrm{H}_{2} \mathrm{O}$ to $55 \mathrm{~cm} \mathrm{H}_{2} \mathrm{O}$. Of 58 with documented pre-operative funduscopy, 42 had papilledema. Of 39 who had documented visual field testing, 25 had pre-operative field defects. The majority of patients had had RGB $(89 \%)$ with a minority undergoing gastroplasty $(6 \%)$ or gastric banding (5\%). Five patients did not experience any improvement in their symptoms but the remainder did. Only 1 of 35 patients with pre-operative papilledema and a postoperative eye exam did not demonstrate resolution of the papilledema. The earliest documented resolution of papilledema occurred at 6 months postoperatively. All of the 12 patients with pre-operative visual field testing who also had postoperative visual field testing demonstrated stabilization or improvement in their visual fields. The authors noted that this remains class IV evidence for treatment of IIH with bariatric surgery and stressed the possibility of publication bias, meaning that case reports and series would be skewed towards those with positive outcomes. 
When treating IIH, alternative treatments including direct medical/surgical management of the IIH should be considered, as well as non-surgical approaches to weight loss. Speed, complications, and effectiveness should all be considered when making individual treatment recommendations.[12]

\section{Weight-associated diseases}

Recent guidelines on obesity management indicate that, in concert with weight loss following successful surgery, diabetic markers decrease, diabetic remissions increase, and diabetic incidence decreases. Similarly, the use of blood pressure medication decreases, hypertension remission increases, cholesterol levels improve, and health-related quality of life improves. All of these effects are found, both within subjects and in comparison to subjects undergoing non-surgical treatments for weight loss.[1] There may even be a mortality benefit in persons undergoing bariatric surgery compared with obese subjects who did not undergo surgery.[8, 13] Additional trials published since the 2013 guidelines provide further evidence regarding the association of bariatric surgery with improvement in weightassociated diseases such as diabetes.[13] There may be some variation in the degree of improvement based on the type of procedure - malabsorption procedures generally giving better results than purely restrictive procedures - though the strength of evidence for this is low.

In contrast to the long-term improvements seen in weight-associated metabolic diseases, such as diabetes, following bariatric surgery, there may be negative effects in the short term, particularly with regard to diabetic complications. Relevant to neuro-ophthalmologists is the recent evidence that short-term improvements in glycemic control may be associated with paradoxical worsening of diabetic retinopathy (DR). A meta-analysis of four case series with 148 patients found that $7.5 \%$ of patients developed DR following bariatric surgery, $57.4 \%$ of patients with DR pre-operatively had no change, $19.2 \%$ of patients had improvement and 23.5\% of patients had progression.[14] Murphy et al reported similar findings in their retrospective study of over 300 diabetic patients who underwent bariatric surgery.[15] 73\% had no change in DR, $11 \%$ had regression and $16 \%$ had progression of DR following bariatric surgery. Male gender, higher level pre-operative DR grade and larger magnitude of postoperative HbA1c reduction were associated with higher-level postoperative DR grade.

\section{Medication pharmakokinetics}

Following bariatric surgery, drug pharmacokinetics are potentially altered via the same mechanisms that reduce nutrient absorption. Potential mechanisms include altered breakdown of drug salts resulting from exposure to a different $\mathrm{pH}$, altered first-pass metabolism due to bypass of certain intestinal components, and altered processing of extended-release pharmaceutical products. This has not been comprehensively studied. Some examples include decreased phenytoin levels and increased atorvastatin levels following bypass-type surgeries.[16] Monitoring therapeutic effects and drug levels (where possible) following bariatric surgery is advised. The use of extended-release or sustainedrelease drug formulations is not advised in patients who have had bariatric surgery, particularly those of the bypass variety. As of September 2014, Pubmed did not list studies examining common neuroophthalmic medications (acetazolamide, topiramate, prednisone). 
NSAIDs should be avoided in patients with restrictive type procedures due to potential direct irritation of the gastric lining.[17]

\section{Nutrition}

Vitamin and mineral absorption is altered following bariatric surgery via the same mechanisms that reduce caloric absorption. Contributing factors include maldigestion, malabsorption and intake deficiency (for example, decreased fat intake as recommended following bypass surgery). Though the literature describing and studying laboratory deficiencies is fairly comprehensive, that dealing with symptomatic deficiencies is less robust.

Laboratory abnormalities-Interestingly, many patients have vitamin and mineral deficiencies prior to bariatric surgery. One study of 232 patients found over $48 \%$ had a deficiency of at least one micronutrient preoperatively, with vitamin $\mathrm{B}_{12}$, zinc, vitamin $\mathrm{D}$ and selenium being the most common.[18] Another study of 267 patients found Vitamin D, A, B12 and iron to be deficient in over 5\% of patients.[19] Zinc and selenium were deficient pre-operatively in less than $5 \%$ of patients. Following surgery, nutrient deficiencies are more common in patients with a bypass component to their operation. $[9,20]$ The type of bypass surgical intervention also has an effect. A study of 141 patients following bariatric surgery found copper deficiency in 50.6\% of BPD patients, but only 3.8\% of RGB patients.[21] Zinc deficiency was also more common in the BPD group.

Though multivitamins are routinely recommended following surgery, these are often inadequate to prevent laboratory deficiencies. Gasteyger et al. found that, of 137 patients who had undergone RGB and who were taking a standard multivitamin preparation, 98\% required additional nutritional supplements by 2 years postoperatively.[22] The most common deficiencies were vitamin $B_{12}$, iron, vitamin $D$ (in $>50 \%$ of patients), folic acid ( $45 \%$ of patients), zinc, vitamin $\mathrm{B}_{6}$, magnesium (12-13\% of patients) and vitamin $\mathrm{B}_{1}$ (4\% of patients). This study excluded patients with pre-operative deficiencies and those noncompliant with follow up. Brolin et al. have suggested that some deficiencies, such as iron, are poorly remediated with oral supplementation.[23] In contrast to these reports, Papamargaritis et al noted no significant change in the prevalence of copper, zinc and selenium surgery pre versus post-operatively in 437 patients taking multivitamin-mineral supplements.[24]

Symptomatic deficiencies-Despite the high incidence of laboratory nutrient deficiencies, and the well-described neurological consequences of vitamin and mineral deficiencies[25],[26-29] (Table 1), the majority of laboratory nutrient deficiencies following bariatric surgery seem to be asymptomatic. Brolin et al. reported in their study of 348 patients who had undergone RGB, including $37 \%$ with laboratory-proven vitamin $\mathrm{B}_{12}$ deficiency, that no patient was symptomatic.[23] In a report of 141 patients following RGB procedures with a high incidence of copper and zinc deficiency on the basis of blood levels, none had neurological symptoms.[21]

Information regarding prevalence of symptomatic vitamin deficiencies following bariatric surgery is limited. Thaisetthawatkul et al. examined neurological complications following 
bariatric surgery, with a focus on peripheral neuropathy.[30] Based on medical record review at a single institution they found that $16 \%$ of patients developed symptomatic, electrodiagnostically-confirmed, peripheral neuropathy after bariatric surgery, compared with $4 \%$ of patients following cholecystectomy. Features of jejuno-ileal bypass surgery (no longer performed) and prolonged nausea/vomiting/dumping/diarrhea were associated with neuropathy development in patients having undergone bariatric surgery, while vitamin $\mathrm{B}_{12}$ injections, multi-vitamin supplementation, calcium supplementation and attendance at a nutritional clinic were inversely associated with neuropathy development in patients having undergone bariatric surgery.

Even less data is available regarding prevalence of neuro-ophthalmic symptoms following bariatric surgery. In Abarbanel et al's prospective series of 500 neurologically normal patients followed over 20 months following Roux-en-Y gastric bypass $(n=457)$ and gastroplasty $(n=43)$ neurologic complications were noted in 23 patients.[31] Diagnosis of complications was based on neurological examinations and testing prompted by patient complaint. One had acute severe neuropathy, electrical myotonia, nystagmus and facial diplegia, one had a myotonic syndrome, two each had burning foot syndrome, WernickeKorsakoff encephalopathy, and posterolateral myelopathy, three had meralgia paresthetica and twelve had symmetric polyneuropathy. This corresponds to a roughly $0.8 \%$ incidence of neuro-ophthalmic presentations (versus 3.8\% other neurologic presentation) in 20 months after surgery. A report of 90 patients attributed a staggering 40-70\% prevalence of night blindness symptoms 6 months after RGB to vitamin A deficiency.[32] However, this study did not include examination findings or visual function testing sufficient to confirm the etiology of these symptoms, so it is difficult to conclude that this is the true prevalence. Though there are many case reports and series that demonstrate the variety of neuroophthalmic presentations of symptomatic vitamin deficiency following bariatric surgery, incidence and prevalence can not be determined from these.

The reasons for why only some patients develop symptomatic vitamin deficiency and many remain asymptomatic are unknown. Case reports of neuro-ophthalmic syndromes post bariatric surgery offer some insight. Multiple concurrent nutrient deficiencies are reported in many symptomatic patients, suggesting that the aggregate effect may be stronger than individual effects.[33, 34] Zinc excess is well known to potentiate copper deficiency and this likely contributed symptomatic copper deficiency in one reported gastric bypass patient who used a zinc containing denture cream.[35] Comorbid non-nutritional risk factors may lower the threshold for vitamin deficiencies to become symptomatic, as demonstrated in a report of a patient found to have post bariatric surgery optic neuropathy with both vitamin deficiency and a Leber hereditary optic neuropathy 11778 mutation.[36] CSF oligoclonal bands were noted in conjunction with nutritional deficiency in 2 patients with optic neuropathy and an additional 2 patients with myelopathy 6 months to 6 years following bariatric surgery.[37] The CSF oligoclonal bands may represent comorbid disease or may be a marker of vitamin deficiency associate inflammation that promotes symptom development.

Neuro-ophthalmic syndromes-The best-characterized syndrome relevant to neuroophthalmology is that of Wernicke's encephalopathy related to thiamine (vitamin $\mathrm{B}_{1}$ ) deficiency, the body stores of which can be depleted in a matter of weeks. In 2007 Singh et 
al. surveyed the literature and identified 37 cases of Wernicke's encephalopathy occurring after bariatric surgery.[38] 21 of these had the complete triad of confusion, ataxia and nystagmus. 7 lacked confusion, 4 lacked nystagmus and only 1 lacked ataxia. 1 patient only had nystagmus. Onset occurred between 2 and 78 weeks after various bariatric surgical procedures, with most presenting within 12 weeks. Many cases were associated with other neurological symptoms, most commonly sensory neuropathy. In addition three cases had third nerve palsies, two had sixth nerve palsies and two had papilledema. The diagnosis was clinical in the majority of cases, with thiamine levels being reported in only 6 patients (and low in only 4 of those). Erythrocyte transketolase levels were reported in 2 patients (but were low in only 1), thus highlighting that thiamine serum levels do not reflect body stores of a nutrient. Following repletion, approximately half of the cases had recovery. These observations reinforce the importance of vigilance for thiamine depletion as a cause of a wide range of neurological symptoms following bariatric surgery and the importance of effectiveness of empiric therapy even in the absence of laboratory confirmation of deficiency.

Optic neuropathy can be caused by copper, B12 and/or thiamine deficiency following bariatric surgery. Thiamine deficiency, discussed above, typically presents in weeks to months following surgery with other associated symptoms. In contrast B12 deficiency typically presents over 1 year following surgery[37] and copper deficiency typically presents over 3 years (and up to over 20 years) after surgery.[39] The reasons for this delay is that it takes years to deplete body stores of these nutrients. Both B12 and copper deficiency induced optic neuropathies can recover with treatment. Therefore it is important to screen for deficiency of both nutrients and replete deficiencies in symptomatic patients. In patients with suspected copper deficiency it is also important to screen for zinc excess, which can be caused by use of zinc containing compounds such as denture cream, as this can potentiate copper deficiency states.[35]

Other neuro-ophthalmic presentations are nyctalopia due to vitamin A and/or zinc deficiency,[32] and ophthalmoparesis due to vitamin E deficiency. In addition many other neurological and ophthalmic symptoms and signs can occur as a result of vitamin deficiency(Table 1). These can present years after surgery, due to the time needed to deplete body stores. Delayed deficiency can also result from late non-compliance with nutritional supplements. Due to the variety of presentations and frequent overlap of multiple deficiencies, it is important for the neuro-ophthalmologist to maintain a broad differential diagnosis in patients who have previously had bariatric surgery, even in the distant past.

\section{Conclusions}

Bariatric surgery is a safe and effective treatment for weight loss in obese individuals. There is level I evidence that is associated with improvement in common weight-associated comorbidities such as diabetes and hypertension and level IV evidence that it is associated with improvement in IIH. Bariatric surgery does not preclude non-surgical weight loss treatment or directed treatment of comorbid conditions including IIH. Guidelines for selection of patients, pre-operative and post-operative management are published regularly by professional organizations and are an excellent resource for the non-bariatric specialist. 
Laboratory nutrient deficiencies following bariatric surgery are common and associated with the type of procedure performed and the type of nutrient supplementation. Clinical syndromes of nutrient deficiency following bariatric surgery are relatively rare and definitive diagnosis can be challenging due to limitations of laboratory testing, overlap of clinical presentations, and coincident conditions.

\section{Acknowledgments}

Funding: K23 EY 024345, Unrestricted Departmental Grant from Research to Prevent Blindness

\section{References}

1. Executive summary: Guidelines (2013) for the management of overweight and obesity in adults: a report of the American College of Cardiology/American Heart Association Task Force on Practice Guidelines and the Obesity Society published by the Obesity Society and American College of Cardiology/American Heart Association Task Force on Practice Guidelines. Based on a systematic review from the The Obesity Expert Panel, 2013. Obesity. 2014; 22:S5-S39. [PubMed: 24961825]

2. Oria HE, Moorehead MK. Bariatric analysis and reporting outcome system (BAROS). Obes Surg. 1998; 8:487-499. [PubMed: 9819079]

3. Ali MR, Maguire MB, Wolfe BM. Assessment of obesity-related comorbidities: a novel scheme for evaluating bariatric surgical patients. Journal of the American College of Surgeons. 2006; 202:7077. [PubMed: 16377499]

4. Krispel CM, Keltner JL, Smith W, Chu DG, Ali MR. Undiagnosed papilledema in a morbidly obese patient population: a prospective study. J Neuroophthalmol. 2011; 31:310-315. [PubMed: 21799447]

5. Hamdallah IN, Shamseddeen HN, Getty JL, Smith W, Ali MR. Greater than expected prevalence of pseudotumor cerebri: a prospective study. Surg Obes Relat Dis. 2013; 9:77-82. [PubMed: 22264908]

6. Raoof N, Sharrack B, Pepper IM, Hickman SJ. The incidence and prevalence of idiopathic intracranial hypertension in Sheffield, UK. Eur J Neurol. 2011; 18:1266-1268. [PubMed: 21426442]

7. American Society for Metabolic and Bariatric Surgery. [cited 2014 August 10] Available from: https://asmbs.org/patients/bariatric-surgery-procedures

8. Mechanick JI, Youdim A, Jones DB, Timothy Garvey W, Hurley DL, Molly McMahon M, Heinberg LJ, Kushner R, Adams TD, Shikora S, Dixon JB, Brethauer S. Clinical practice guidelines for the perioperative nutritional, metabolic, and nonsurgical support of the bariatric surgery patient--2013 update: cosponsored by American Association of Clinical Endocrinologists, the Obesity Society, and American Society for Metabolic \& Bariatric Surgery. Surg Obes Relat Dis. 2013; 9:159-191. [PubMed: 23537696]

9. Gudzune KA, Huizinga MM, Chang HY, Asamoah V, Gadgil M, Clark JM. Screening and diagnosis of micronutrient deficiencies before and after bariatric surgery. Obes Surg. 2013; 23:1581-1589. [PubMed: 23515975]

10. Brolin RE, Leung M. Survey of vitamin and mineral supplementation after gastric bypass and biliopancreatic diversion for morbid obesity. Obes Surg. 1999; 9:150-154. [PubMed: 10340768]

11. Fridley J, Foroozan R, Sherman V, Brandt ML, Yoshor D. Bariatric surgery for the treatment of idiopathic intracranial hypertension. J Neurosurg. 2011; 114:34-39. [PubMed: 20095788]

12. Banik R. Obesity and the role of nonsurgical and surgical weight reduction in idiopathic intracranial hypertension. Int Ophthalmol Clin. 2014; 54:27-41. [PubMed: 24296369]

13. Arterburn DE, Olsen MK, Smith VA, Livingston EH, Van Scoyoc L, Yancy WS Jr. Eid G, Weidenbacher H, Maciejewski ML. Association between bariatric surgery and long-term survival. Jama. 2015; 313:62-70. [PubMed: 25562267] 
14. Cheung D, Switzer NJ, Ehmann D, Rudnisky C, Shi X, Karmali S. The Impact of Bariatric Surgery on Diabetic Retinopathy: A Systematic Review and Meta-Analysis. Obes Surg. 2015; 25:16041609. [PubMed: 25515499]

15. Murphy R, Jiang Y, Booth M, Babor R, MacCormick A, Hammodat H, Beban G, Barnes RM, Vincent AL. Progression of diabetic retinopathy after bariatric surgery. Diabet Med. 2015

16. Sawaya RA, Jaffe J, Friedenberg L, Friedenberg FK. Vitamin, mineral, and drug absorption following bariatric surgery. Curr Drug Metab. 2012; 13:1345-1355. [PubMed: 22746302]

17. Titus R, Kastenmeier A, Otterson MF. Consequences of gastrointestinal surgery on drug absorption. Nutr Clin Pract. 2013; 28:429-436. [PubMed: 23835364]

18. Ernst B, Thurnheer M, Schmid SM, Schultes B. Evidence for the necessity to systematically assess micronutrient status prior to bariatric surgery. Obes Surg. 2009; 19:66-73. [PubMed: 18491197]

19. Lefebvre P, Letois F, Sultan A, Nocca D, Mura T, Galtier F. Nutrient deficiencies in patients with obesity considering bariatric surgery: a cross-sectional study. Surg Obes Relat Dis. 2014; 10:540 546. [PubMed: 24630922]

20. Becker DA, Balcer LJ, Galetta SL. The Neurological Complications of Nutritional Deficiency following Bariatric Surgery. J Obes. 2012; 20128 pages.

21. Balsa JA, Botella-Carretero JI, Gomez-Martin JM, Peromingo R, Arrieta F, Santiuste C, Zamarron I, Vazquez C. Copper and zinc serum levels after derivative bariatric surgery: differences between Roux-en-Y Gastric bypass and biliopancreatic diversion. Obes Surg. 2011; 21:744-750. [PubMed: 21442375]

22. Gasteyger C, Suter M, Gaillard RC, Giusti V. Nutritional deficiencies after Roux-en-Y gastric bypass for morbid obesity often cannot be prevented by standard multivitamin supplementation. Am J Clin Nutr. 2008; 87:1128-1133. [PubMed: 18469230]

23. Brolin RE, Gorman JH, Gorman RC, Petschenik AJ, Bradley LJ, Kenler HA, Cody RP. Are vitamin B12 and folate deficiency clinically important after roux-en-Y gastric bypass? J Gastrointest Surg. 1998; 2:436-442. [PubMed: 9843603]

24. Papamargaritis D, Aasheim ET, Sampson B, le Roux CW. Copper, selenium and zinc levels after bariatric surgery in patients recommended to take multivitamin-mineral supplementation. J Trace Elem Med Biol. 2015; 31:167-172. [PubMed: 25271186]

25. Kumar N. Neurological Complications of Bariatric Surgery in Neurology of Systemic Disease. Continuum. 2014; 20:580-597. [PubMed: 24893235]

26. Pazirandeh, S.; Lo, C.; Burns, D. Overview of Water Soluble Vitamins. Lipman, TO., editor. UpToDate; Waltham, MA: 2014. [Accessed on August 10, 2015]

27. Pazirandeh, S.; Burns, D.; Griffin, I. Overview of dietary trace minerals. Lipman, TO., editor. UpToDate; Waltham, MA: 2014. [Accessed on August 10, 2015]

28. Pazirandeh, S.; Burns, D. Overview of vitamin A. Lipman, TO.; Motil, KJ., editors. UpToDate; Waltham, MA: 2015. [Accessed on August 10, 2015]

29. Traber MG. Vitamin E inadequacy in humans: causes and consequences. Adv Nutr. 2014; 5:503514. [PubMed: 25469382]

30. Thaisetthawatkul P, Collazo-Clavell ML, Sarr MG, Norell JE, Dyck PJB. A controlled study of peripheral neuropathy after bariatric surgery. Neurology. 2004; 63:1462-1470. [PubMed: 15505166]

31. Abarbanel JM, Berginer VM, Osimani A, Solomon H, Charuzi I. Neurologic complications after gastric restriction surgery for morbid obesity. Neurology. 1987; 37:196-196. [PubMed: 3027610]

32. Pereira S, Saboya C, Ramalho A. Impact of different protocols of nutritional supplements on the status of vitamin A in class III obese patients after Roux-en-Y gastric bypass. Obes Surg. 2013; 23:1244-1251. [PubMed: 23462856]

33. Ramos-Levi AM, Sanchez-Pernaute A, Rubio Herrera MA. Dermatitis and optic neuropathy due to zinc deficiency after malabsortive bariatric surgery. Nutr Hosp. 2013; 28:1345-1347. [PubMed: 23889664]

34. Stroh C, Weiher C, Hohmann U, Meyer F, Lippert H, Manger T. Vitamin A deficiency (VAD) after a duodenal switch procedure: a case report. Obes Surg. 2010; 20:397-400. [PubMed: 19847574] 
35. Yarandi SS, Griffith DP, Sharma R, Mohan A, Zhao VM, Ziegler TR. Optic Neuropathy, Myelopathy, Anemia, and Neutropenia Caused by Acquired Copper Deficiency After Gastric Bypass Surgery. J Clin Gastroenterol. 2014; 48:862-865. [PubMed: 24583748]

36. Santos-Garcia D, Abella J, De Domingo B, de la Fuente-Fernandez R. Leber hereditary optic neuropathy associated with malabsorption syndrome after bariatric surgery. J Neuroophthalmol. 2009; 29:75-76. [PubMed: 19458584]

37. Juhasz-Pocsine K, Rudnicki SA, Archer RL, Harik SI. Neurologic complications of gastric bypass surgery for morbid obesity. Neurology. 2007; 68:1843-1850. [PubMed: 17515548]

38. Singh S, Kumar A. Wernicke encephalopathy after obesity surgery: a systematic review. Neurology. 2007; 68:807-811. [PubMed: 17353468]

39. Shah AR, Tamhankar MA. Optic neuropathy associated with copper deficiency after gastric bypass surgery. Retin Cases Brief Rep. 2014; 8:73-76. [PubMed: 25372214] 

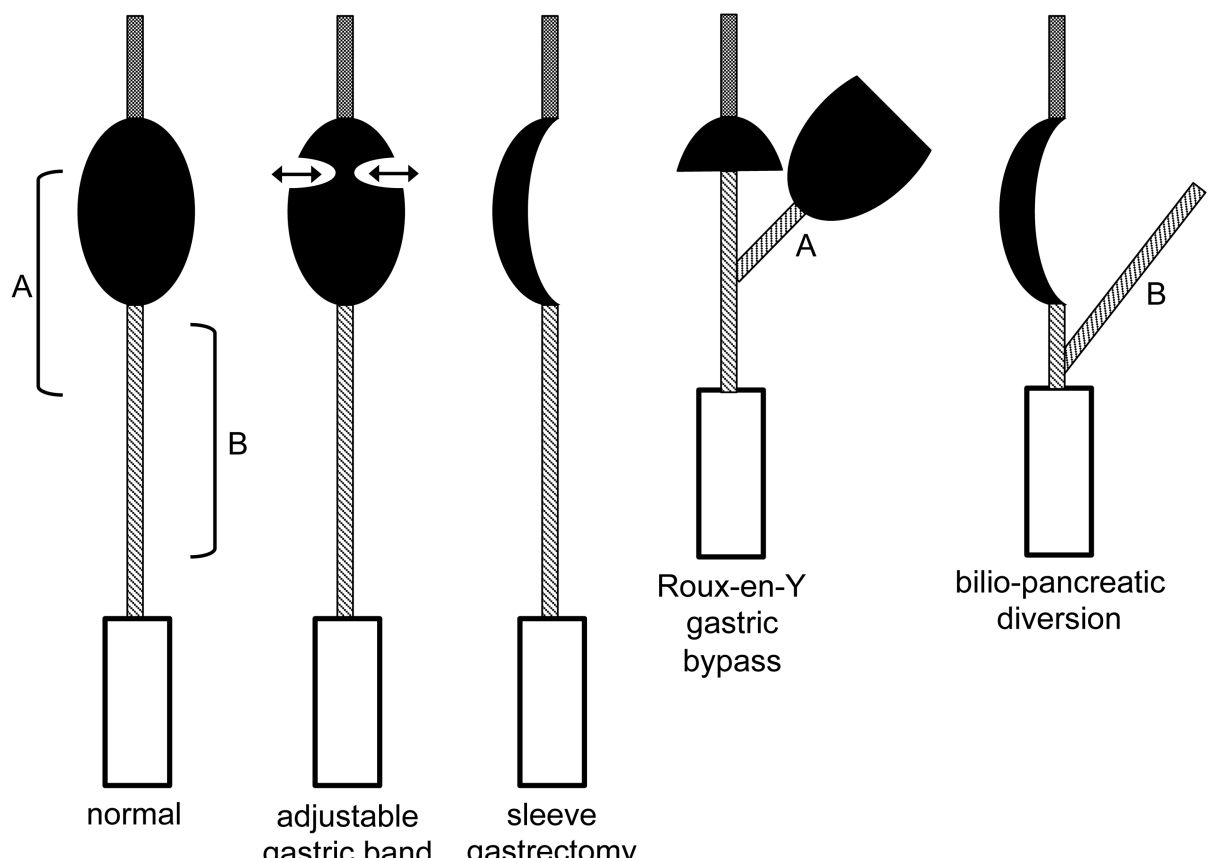

gastric

bypass

Figure 1. Bariatric Surgery Procedures

schematic of gastrointestinal tract showing esophagus (grey rectangle), stomach (black oval), small intestine (striped rectangle) and large intestine (white rectangle). Normal anatomy is illustrated on the left. Other illustrations show common bariatric surgical interventions. A and B indicate bypassed segments in Roux-en-Y and biliopancreatic diversion respectively. 


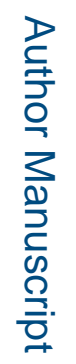

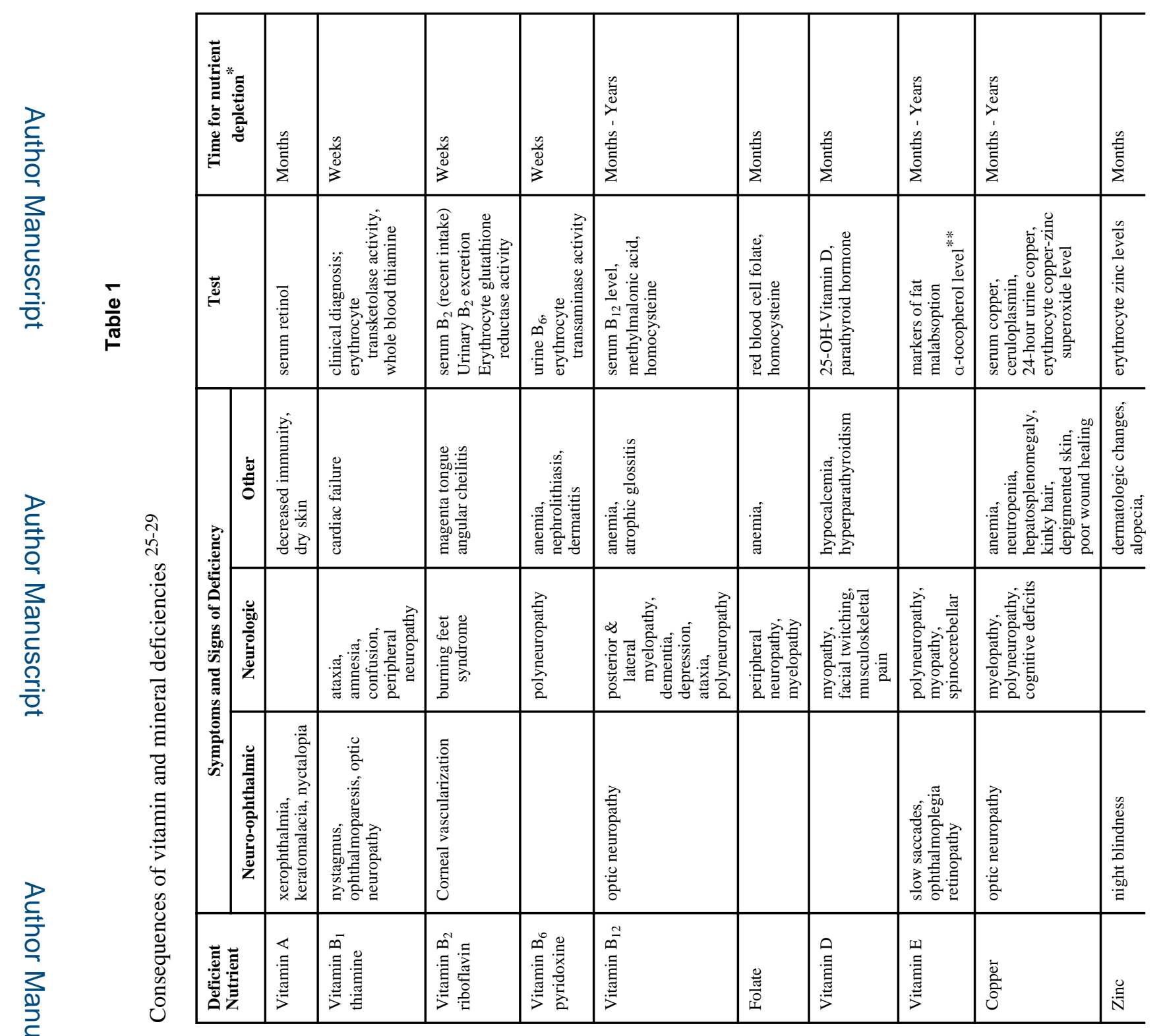

J Neuroophthalmol. Author manuscript; available in PMC 2017 March 01. 

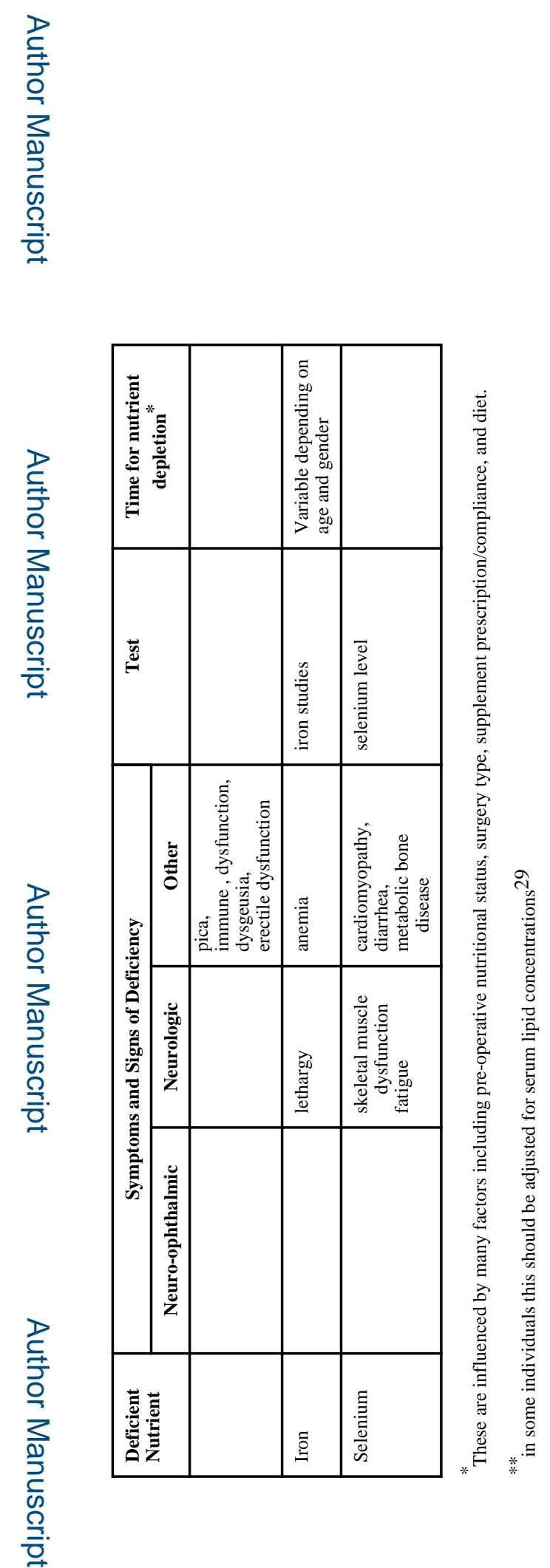

J Neuroophthalmol. Author manuscript; available in PMC 2017 March 01. 